\title{
A Close Examination of CMB Mirror-Parity
}

\author{
Assaf Ben-David ${ }^{1}$ and Ely D. Kovetz ${ }^{2}$ \\ ${ }^{1}$ Niels Bohr International Academy and Discovery Center, The Niels Bohr Institute, \\ Blegdamsvej 17, DK-2100 Copenhagen $\varnothing$, Denmark; email: bendavid@nbi.dk \\ ${ }^{2}$ Theory Group, Department of Physics and Texas Cosmology Center, \\ The University of Texas at Austin, TX 78712, USA; email: elykovetz@gmail.com
}

\begin{abstract}
We revisit recent claims of significant mirror-parity in CMB data from WMAP and Planck with a careful analysis using statistical estimators in both harmonic and pixel spaces. While the data indeed show significant signs of odd mirror-parity under some circumstances, the broad study shows that the results are not in significant tension with $\Lambda \mathrm{CDM}$.
\end{abstract}

Keywords. cosmic microwave background, methods: data analysis, methods: statistical

\section{Method and Data}

Pixel-Based Statistic: Under reflection through the plane normal to $\hat{\mathbf{n}}$, a direction $\hat{\mathbf{r}}$ in the sky transforms as $\hat{\mathbf{r}} \rightarrow \hat{\mathbf{r}}_{\hat{\mathbf{n}}}=\hat{\mathbf{r}}-2(\hat{\mathbf{r}} \cdot \hat{\mathbf{n}}) \hat{\mathbf{n}}$. The score for mirror-parity in pixel-space is the temperature difference between hemispheres, averaged over all unmasked spatial directions (de Oliveira-Costa et al. 2004; Finelli et al. 2012; Ade et al. 2013b; Ben-David \& Kovetz 2014)

$$
S_{\mathrm{p}}^{ \pm}(\hat{\mathbf{n}})=\overline{\left[\frac{T(\hat{\mathbf{r}}) \pm T\left(\hat{\mathbf{r}}_{\hat{\mathbf{n}}}\right)}{2}\right]^{2}} .
$$

With this statistic, a large degree of even (odd) mirror-parity is given by a low $S_{\mathrm{p}}^{-}\left(S_{\mathrm{p}}^{+}\right)$. To apply a Galactic mask, the masked pixels are simply ignored in the summation.

Harmonic Statistic: Under reflection through the $z$-axis, $Y_{\ell m}(\hat{\mathbf{r}}) \rightarrow(-1)^{\ell+m} Y_{\ell m}(\hat{\mathbf{r}})$. The harmonic parity score compares for each symmetry-plane direction the relative power of even and odd multipoles (Ben-David et al. 2012; Ben-David \& Kovetz 2014)

$$
S_{\mathrm{h}}(\hat{\mathbf{n}})=\sum_{\ell=2}^{\ell_{\max }} \sum_{m=-\ell}^{\ell}(-1)^{\ell+m} \frac{\left|a_{\ell m}(\hat{\mathbf{n}})\right|^{2}}{\widehat{C}_{\ell}}-\left(\ell_{\max }-1\right),
$$

where $\hat{\mathbf{n}}$ is the $z$-axis used in the harmonic expansion and the score is normalized by the actual observed power $\widehat{C}_{\ell}=(2 \ell+1)^{-1} \sum_{m}\left|a_{\ell m}\right|^{2}$. The parameter $\ell_{\max }$ allows to easily test the scale dependence of the results. With this statistic, a large degree of even (odd) mirror-parity is given by a high (low) value for $S_{\mathrm{h}}$. On a masked map a method for maximum likelihood reconstruction of the harmonic coefficients is used.

Significance Estimation: We use two complementing methods: (i) Compare the "best" raw score $R$, i.e. the maximum or minimum of the score map, to random $\Lambda$ CDM realizations (Ade et al. 2013b). (ii) Normalize the "best" raw score as $\bar{R}=|R-\mu| / \sigma$, where $\mu$ and $\sigma$ are the mean and standard deviation of the score-map over all directions (Ben-David et al. 2012), and compare $\bar{R}$ to random $\Lambda$ CDM realizations. While the first method is straightforward, the second also tests whether the anomalous direction is unique. This is suited for a scenario in which the anomaly is of a cosmological origin, and a single anomalous direction is expected. We use a set of $10^{4}$ random $\Lambda$ CDM realizations using the best-fit power-spectrum. We mask randoms and the data with the same mask. 


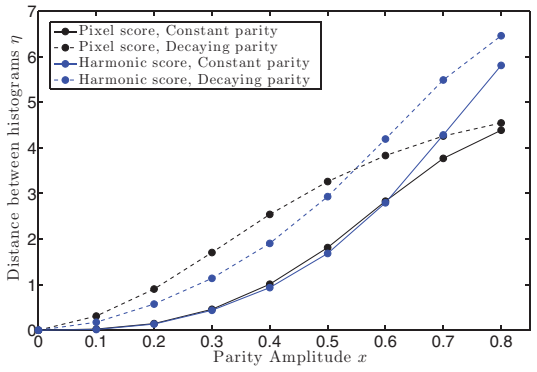

(a)

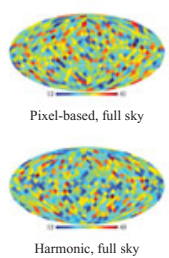

Harmonic, full sky

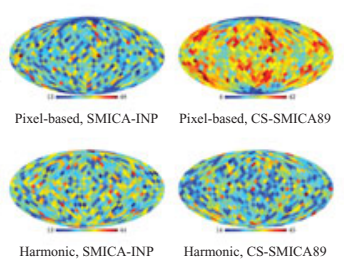

(b)

Figure 1. (a) Estimate of the signal-to-noise ratio for the detection of mirror-parity as a function of the parity modulation amplitude. (b) Histograms of the direction of maximal parity for random realization with the various masks.

Data: We use the Planck SMICA and NILC maps (Ade et al. 2013a). We also use the LGMCA map released by Bobin et al. (2014). This map is a combination of data from WMAP and Planck that is claimed to be a rather clean full sky map. We also use three Galactic masks in our analysis (Ade et al. 2013a): the large unified U73 mask and the confidence and inpainting masks of the SMICA map, CS-SMICA89 and SMICA-INP. These masks have $f_{\text {sky }}=0.73,0.89$ and 0.97 , respectively. We smooth all maps and masks with a Gaussian beam with FWHM of $\sim 11^{\circ}$ and degrade them to $N_{\text {side }}=16$.

\section{Tests on Random Simulations}

We test our parity statistics on random simulations to assess their effectiveness.

Detectability of Mirror-Parity: Fig. 1(a) shows a rough estimate of the $\mathrm{S} / \mathrm{N}$ for the detection of mirror-parity using the two statistics. We used random $\Lambda$ CDM realizations modulated to contain traces of even mirror-parity and calculated the distances between the histograms for the modulated randoms and the unmodulated ones. The modulation amplitude is either constant or exponentially decaying with scale. Both statistics are effective in detecting mirror-parity, and perform similarly in this test.

Sensitivity to the Total Power: The theoretical power spectrum on large scales is not well determined. The power observed on these scales is somewhat low compared with expectations. How sensitive are the parity statistics to this uncertainty? The harmonic statistic is manifestly normalized by the total observed power, whereas the pixel-based statistic is not. We tested parity-modulated randoms and calculated the significance of the results once versus normal $\Lambda \mathrm{CDM}$ realizations and then versus realizations constrained to have low power, consistent with the levels measured by Planck. We find that when comparing to the "wrong" ensemble, the pixel-based significance changes by $\sim 0.5 \sigma$. The harmonic significance level is, however, much more stable and only changes by $\sim 0.02 \sigma$.

Bias on Masked Sky: We find the direction exhibiting maximal even parity for each of the isotropic $\Lambda$ CDM realizations. We repeat the test using each of the masks, as well as using the full sky. In Fig. 1(b) we plot histograms of the results. Since the randoms are isotropic, a non-uniform distribution can only be attributed to the non-isotropic mask. While both statistics are isotropic on the full sky, as the masked area is increased the pixel-based statistic is increasingly biased, while the harmonic-statistic is unaffected. 


\section{Results}

For all map and mask combinations and for both statistics, we identify a single direction of maximal even mirror-parity and a corresponding one for odd mirror-parity.

Even Mirror-Parity Direction $(l, b) \sim\left(260^{\circ}, 48^{\circ}\right)$ : Regardless of which map and mask are considered, which statistic and which significance estimator, the significance level for the even parity direction never reaches $3 \sigma$ and is therefore not anomalous.

Odd Mirror-Parity Direction $(l, b) \sim\left(264^{\circ}, 17^{\circ}\right)$ : The significance levels of the odd parity direction using both the $R$ and $\bar{R}$ estimators range from $1.5-3 \sigma$. When masked with the large U73 mask and measured using the $R$ estimator, the results are marginally significant, crossing the $3 \sigma$ level (matching Ade et al. 2013b). However, with a smaller mask or no mask, the results are insignificant. When measured using the $\bar{R}$ estimator, the results are not significant for any of the masks. The significance levels for the harmonic statistic are not high either. No combination of map and mask shows a significant $(>3 \sigma)$ level of odd parity regardless of the significance estimator used, for any $5 \leqslant \ell_{\max } \leqslant 9$.

\section{Conclusion}

We have reproduced the results of Ade et al. (2013b) showing a marginally significant $(\sim 3 \sigma)$ odd parity direction in the Planck data using a pixel-based statistic and the large U73 mask. However, we have shown that these results are biased due to the large mask, and are also sensitive to assumptions regarding the total power on large scales, which is weakly constrained by the data. Indeed, with a smaller mask, the significance level is much lower.

Using a harmonic statistic, which we have shown to be far more robust, the odd-parity direction is not significant, no matter the map and mask used, for any of the tested large scales $\left(5 \leqslant \ell_{\max } \leqslant 9\right)$.

In light of these findings, we conclude that while there is some tendency for odd parity in the CMB data, when embracing a broader perspective and examining the complete set of data maps and Galactic masks and the properties of the statistical estimators, it appears that the evidence for anomalous mirror-parity is rather weak. It poses no real challenge to the concordance model, and should therefore not be considered a $\Lambda \mathrm{CDM}$ anomaly.

\section{Acknowledgements}

ABD was supported by the Danish National Research Foundation. EDK was supported by the National Science Foundation under Grant Number PHY-1316033.

\section{References}

Ade P. A. R., et al. (The Planck Collaboration) 2013a, preprint (arXiv:1303.5072)

Ade P. A. R., et al. (The Planck Collaboration) 2013b, preprint (arXiv:1303.5083)

Ben-David A., Kovetz E. D., \& Itzhaki N. 2012, ApJ, 748, 39

Ben-David A. \& Kovetz E. D. 2014, preprint (arXiv:1403.2104)

Bobin J., Sureau F., Starck J.-L., Rassat A., \& Paykari P. 2014, A\& A, 563, A105

de Oliveira-Costa A., Tegmark M., Zaldarriaga M., \& Hamilton A. 2004, Phys. Rev. D, 69, 063516

Finelli F., Gruppuso A., Paci F., \& Starobinsky A. A. 2012, J. Cosmol. Astropart. Phys., 7, 49 